UNIVERSIDADE DE SÃO PAULO

FACULDADE DE CIÊNCIAS FARMACÊUTICAS DE RIBEIRÃO PRETO

GABRIELA TONINATO DE PAULA

Análises filogenética, morfológica e química de Zygosaccharomyces associados a abelhas sem ferrão 
GABRIELA TONINATO DE PAULA

\section{Análises filogenética, morfológica e química de Zygosaccharomyces associados a abelhas sem ferrão}

Dissertação de Mestrado apresentada ao Programa de Pós-Graduação em Ciências Farmacêuticas da Faculdade de Ciências Farmacêuticas de Ribeirão Preto/USP para obtenção do Título de Mestre em Ciências

Área de Concentração: Produtos Naturais e Sintéticos

Orientadora: Profa. Dra. Mônica Tallarico Pupo

Versão corrigida da Dissertação de Mestrado apresentada ao Programa de Pós-Graduação em Ciências Farmacêuticas no dia 08/04/2020. A versão original encontra-se disponível na Faculdade de Ciências Farmacêuticas de Ribeirão Preto/USP. 


\section{RESUMO}

PAULA, G. T. Análises filogenética, morfológica e química de Zygosaccharomyces associados a abelhas sem ferrão. 2020. 95f. Dissertação (Mestrado). Faculdade de Ciências Farmacêuticas de Ribeirão Preto - Universidade de São Paulo, Ribeirão Preto, 2020.

Insetos sociais são conhecidos por estabelecerem simbiose com micro-organismos. Suas colônias fornecem microambientes adequados para o crescimento microbiano, como condições ambientais estáveis e uma rica fonte de alimento. Em troca, os insetos podem ser beneficiados com nutrientes e/ou proteção contra patógenos. No entanto, pouco se conhece sobre esse tipo de interação mutualística envolvendo abelhas. Apenas recentemente foi descrita uma interação simbiótica entre a abelha sem ferrão Scaptotrigona depilis e o fungo Zygosaccharomyces sp. Esse fungo cresce no interior das células de cria de $S$. depilis e é ingerido pelas larvas, sendo indispensável para a sobrevivência e desenvolvimento das mesmas. Isso se deve à capacidade que o fungo tem de acumular lipídeos em adipossomos, dentre eles o esteroide ergosterol, que é utilizado pelas larvas de $S$. depilis durante seu processo metamórfico. No presente estudo, o objetivo foi verificar se o mesmo fenômeno também ocorre em outras espécies de abelhas sem ferrão. Objetivou-se verificar se há a presença de fungo-alimento simbionte e se o mesmo é fonte de esteroides para o desenvolvimento larval. Para isso, foram obtidas amostras da célula de cria de 19 espécies de abelhas sem ferrão pertencentes a 12 gêneros diferentes. Foi possível isolar Zygosaccharomyces spp. de oito espécies. Além das células de cria também foi possível obter Zygosaccharomyces spp. de outras partes da colônia, como mel, pólen, inseto adulto e refugo/lixo, o que possibilitou fazer análises comparativas. As análises de imagem por microscopia óptica demonstraram que as linhagens de Zygosaccharomyces spp. isoladas das células de cria são capazes de formar pseudo-hifas enquanto os demais isolados de outros locais da colônia apresentam apenas células esféricas e/ou ovóides. Adicionalmente, com os dados do sequenciamento genético das regiões D1/D2 do gene 26S e 18S do rRNA foram obtidas três árvores filogenéticas. Nelas, foi possível observar a formação de um subclado constituído apenas pelas linhagens de Zygosaccharomyces spp. isoladas das células de cria de diferentes espécies de abelhas sem ferrão. Esses resultados ressaltam a especificidade desse fungo para esse sistema envolvendo as abelhas sem ferrão. A análise de esteroides e quantificação de ergosterol através de GC-MS foi realizada para as linhagens identificadas como Zygosaccharomyces spp., isoladas da célula de cria e de outros locais da colônia, como também de outros fungos filamentosos isolados esporadicamente das células de cria de algumas abelhas. Os dados de GC-MS demonstraram que não há um padrão diferenciado na concentração desse esteroide entre as linhagens. Os resultados obtidos indicam que a associação de Zygosaccharomyces parece ser uma interação simbiótica frequente em abelhas sem ferrão. A determinação da associação desses micro-organismos nesse sistema fornece subsídios para estudos adicionais quanto ao uso de fungicidas na agricultura, uma vez que estes podem afetar indiretamente o desenvolvimento dessas abelhas.

Palavras-chave: Abelhas sem ferrão, Zygosaccharomyces, Célula de cria, Ergosterol, Pseudo-hifas. 


\begin{abstract}
PAULA, G. T. Phylogenetic, morphological and chemical analyzes of Zygosaccharomyces associated with stingless bees. 2020. 95f. Dissertation (Master). Faculdade de Ciências Farmacêuticas de Ribeirão Preto - Universidade de São Paulo, Ribeirão Preto, 2020.
\end{abstract}

Social insects are known to establish symbiosis with microorganisms. Their colonies provide microenvironments suitable for microbial growth, such as stable environmental conditions and rich source of food. In return, insects can benefit from nutrients and/or protection against pathogens. However, little is known about this type of mutualistic interaction involving bees. Only recently a symbiotic interaction has been described between the stingless bee Scaptotrigona depilis and the fungus Zygosaccharomyces sp. This fungus grows inside the brood cells of $S$. depilis and is ingested by the larvae, being indispensable for their survival and development. This is due to the fungus' ability to accumulate lipids in adiposomes, including the steroid ergosterol, which is used by S. depilis larvae during their metamorphic process. In the present study, the objective was to verify whether the same phenomenon also occurs in other species of stingless bees. The objective was to verify if there is the presence of symbiotic food fungus and if it is a source of steroids for larval development. For this, samples were obtained from the brood cells of 19 species of stingless bees belonging to 12 different genera. Zygosaccharomyces spp. was isolated from eight species. In addition to the brood cells, it was also possible to obtain Zygosaccharomyces spp. from other parts of the colony, such as honey, pollen, adult insect and refuse/garbage, which made it possible to make comparative analyzes. Image analysis by optical microscopy demonstrated that Zygosaccharomyces spp. strains isolated from brood cells are capable of forming pseudohyphae, while the others isolated from other sites in the colony presented spherical and/or ovoid cells. Genetic sequencing of the D1/D2 regions of the 26S and 18S rRNA gene allowed the construction of three phylogenetic trees. The phylogenetic analyses showed the formation of a clade made up only of Zygosaccharomyces spp. strains isolated from the brood cells of different stingless bee species. These results highlight the specificity of the fungus for this system involving stingless bees. The analysis of steroids and ergosterol quantification by GC-MS was carried out for the strains identified as Zygosaccharomyces spp., isolated from the brood cell and other colony sites, as well as from other filamentous fungi isolated sporadically from the brood cells of some bees. The GC-MS data demonstrated that there is no different pattern in the concentration of this steroid among the strains. Altogether, the results indicate that the association of Zygosaccharomyces seems to be a frequent symbiotic interaction in stingless bees. The determination of the association of these microorganisms in this system provides subsidies for additional studies regarding the use of fungicides in agriculture since they can indirectly affect the development of these bees.

Keywords: stingless bees, Zygosaccharomyces, brood cell, ergosterol, pseudohyphae. 


\section{INTRODUÇÃO}

\subsection{Abelhas sem ferrão}

Os insetos sociais, dentre eles as formigas, cupins e algumas abelhas e vespas, são conhecidos pela sua capacidade de viver em grupos de forma harmônica, capazes de explorar uma grande variedade de recursos, viver em ambientes extremos, além de influenciar na ecologia e evolução de outros ecossistemas (WILSON, 1990). Além disso, são caracterizados pela divisão reprodutiva do trabalho, cuidado com a prole e sobreposição da geração de indivíduos, vivendo em colônias que variam de centenas a milhares de indivíduos (SAKAGAMI, 1982; DANFORTH, 2007; HÖLLDOBLER e WILSON, 2009).

As abelhas sem ferrão pertencem à ordem Hymenoptera, superfamília Apoidea, família Apidae, subfamília Apinae e tribo Meliponini (MICHENER, 2007). Por apresentarem diferenças na morfologia e na origem das rainhas.

Apesar do nome, essas abelhas apresentam ferrão, mas este é atrofiado e não funcional (MICHENER, 2013). Elas também são popularmente conhecidas como abelhas nativas, abelhas indígenas ou até mesmo por meliponíneos (MICHENER, 2007).

Essas abelhas são consideradas como um dos principais polinizadores de vários ecossistemas neotropicais (KERR et al., 1996), onde desempenham um papel importante na polinização de plantas nativas e auxiliam na polinização de lavouras. Café (Coffea arabica e Coffea canephora), abacate (Persea americana), morango (Fragaria ananassa), tomate (Lycopersicon esculentum), pepino (Cucumis sativus), manga (Mangifera indica) são alguns exemplos de culturas efetivamente polinizadas por abelhas sem ferrão (SLAA et al., 2006). Algumas espécies produzem mel, pólen, cera e própolis que são explorados comercialmente (NOGUEIRA-NETO, 1997).

Com uma ampla área de distribuição geográfica, as abelhas nativas habitam grande parte das áreas tropicais do mundo (MICHENER, 2007), bem como as regiões subtropicais do hemisfério sul (SILVEIRA et al., 2002). A tribo Meliponini compreende mais de 500 espécies descritas e muitas ainda não descritas (RASMUSSEN e CAMERON, 2010). No Brasil há uma grande diversidade (Figura 1), pois já foram descritas aproximadamente 243 espécies (CAMARGO e PEDRO, 2013; ASCHER e PICKERING, 2020). 


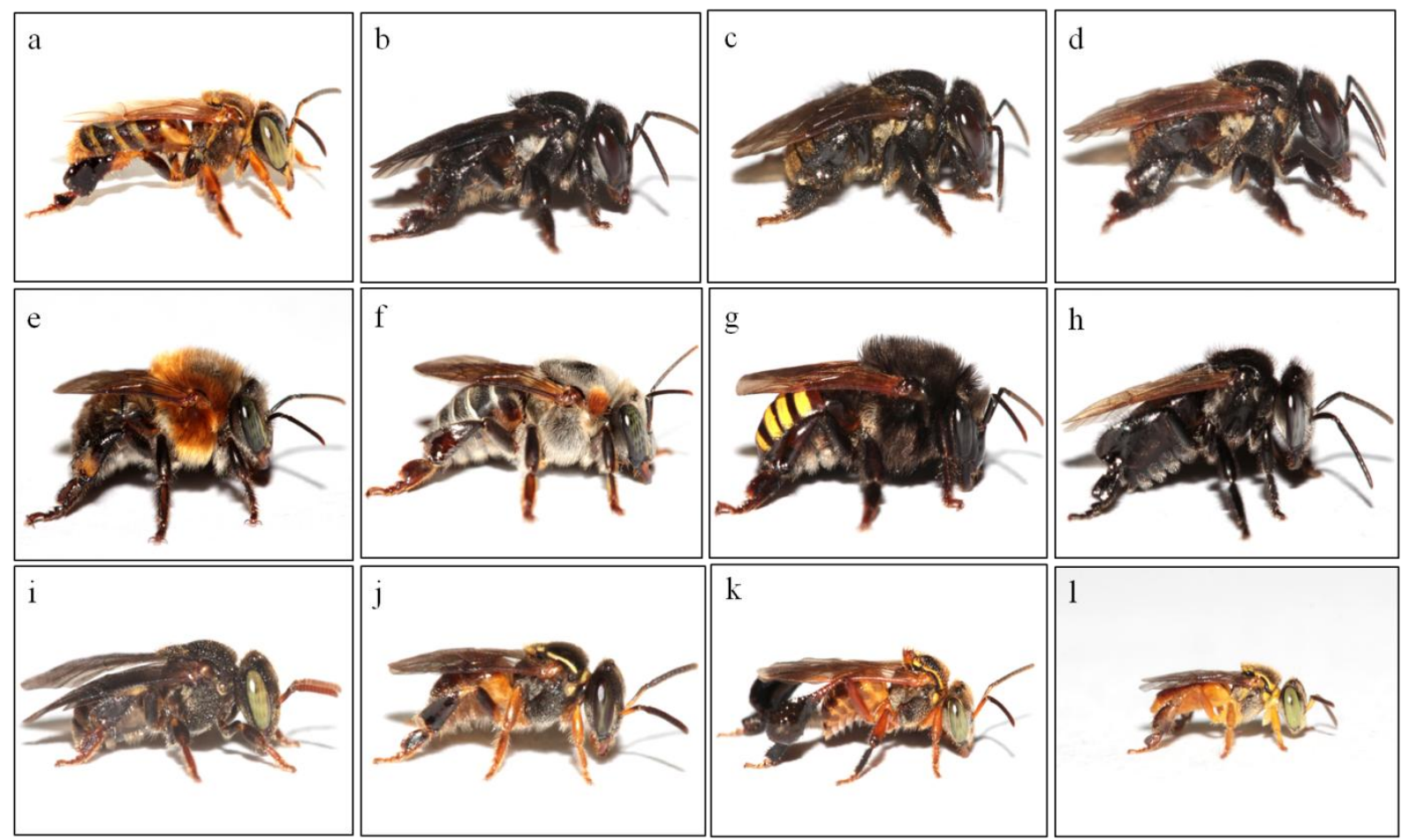

Figura 1. Variedade de espécies de abelhas sem ferrão. (a) Tetragona elongata (Borá). (b) Scaptotrigona bipunctata (Tubuna). (c) S. tubiba (Tubi) (d) S. postica (Mandaguari) (e) Melipona bicolor (Guaraipo) (f) M. fasciculata (Tiúba) (g) M. quadrifasciata (Mandaçaia) (h) Geotrigona mombuca (Mombuca) (i) Nannotrigona testaceicornis (Iraí) (j) Plebeia droryana (Mirim) (k) Frieseomelitta varia (Marmelada) (l) Tetragonisca angustula (Jataí). Todas as imagens: Cristiano Menezes

Os meliponíneos são conhecidos como insetos eussociais por apresentarem divisão reprodutiva do trabalho, sobreposição de gerações e cuidados cooperativos com a ninhada (SAKAGAMI, 1982; DANFORTH, 2007). Essas abelhas, assim como muitos insetos sociais (formigas, cupins e algumas vespas), possuem suas famílias divididas em castas (VENTURIERI, 2008). Na divisão do trabalho reprodutivo, há uma casta responsável apenas pela reprodução e uma casta assimilativa responsável pelas demais funções da colônia (NOGUEIRA-NETO, 1997). A rainha é responsável pela postura dos ovos e coesão da colônia. Já as operárias (ou obreiras) são responsáveis pela maioria dos trabalhos, como limpeza, produção de cera, alimentação da rainha, preenchimento das células com alimento larval, proteção contra inimigos externos, coleta de recursos externos (néctar, pólen, resina, barro e fibra) e eliminação dos detritos da colônia. Os machos participam pouco das atividades da colônia, tendo sua função principal resumida à cópula da rainha durante o voo nupcial (VENTURIERI, 2008).

Ademais, as abelhas sem ferrão apresentam uma grande diversidade morfológica, além da variação no tamanho dos indivíduos (de 2 a $14 \mathrm{~mm}$ ), tamanho da colônia, local do ninho, comportamento, dentre outros (SLAA et al., 2006). As colônias são perenes e podem conter de algumas dezenas a mais de 10.000 indivíduos (MICHENER, 2007). Os ninhos dessas 
abelhas são muito diversificados (VENTURIERI, 2008), no geral construídos em cavidades pré-existentes como ocos de árvores, ninhos abandonados de cupins e formigas ou cavidades escavadas pelas próprias abelhas. Há também algumas espécies que constroem o ninho exposto em galhos de árvores, paredões rochosos ou em paredes de construções humanas (SILVEIRA et al., 2002; MICHENER, 2007). Os mesmos podem ser constituídos por diversos materiais encontrados na natureza como cerume, resinas, barro e até mesmo excrementos. Adicionalmente, a cera que elas produzem também pode ser usada para a construção do ninho.

As principais estruturas encontradas dentro da colônia de Meliponíneos estão representadas na Figura 2. Os batumes são grandes formações de barro misturado à própolis que servem para a vedação de frestas ou delimitação das cavidades onde os Meliponíneos residem. A entrada e o túnel de ingresso, em muitos casos, podem ter formato específico para cada espécie. Há também o invólucro que, geralmente, está em torno dos favos de cria, uma de suas principais funções é proteger e conservar o calor nessa região. Para armazenar seus alimentos as abelhas sem ferrão utilizam estruturas conhecidas como potes de alimentos (NOGUEIRA-NETO, 1997), sendo o mel e o pólen armazenados separadamente (SAKAGAMI et al., 1993). Um dos principais componentes do ninho são as células/favos de cria, estruturas de especial interesse nesse trabalho. As células de cria são preenchidas, na maior parte, com alimento larval, em seguida elas recebem o ovo e, por fim, são fechadas. Durante todo o desenvolvimento da cria (fases de larva e pupa) a célula permanece fechada e somente é reaberta quando o inseto adulto emergir (NOGUEIRA-NETO, 1997; ROUBIK, 2006).
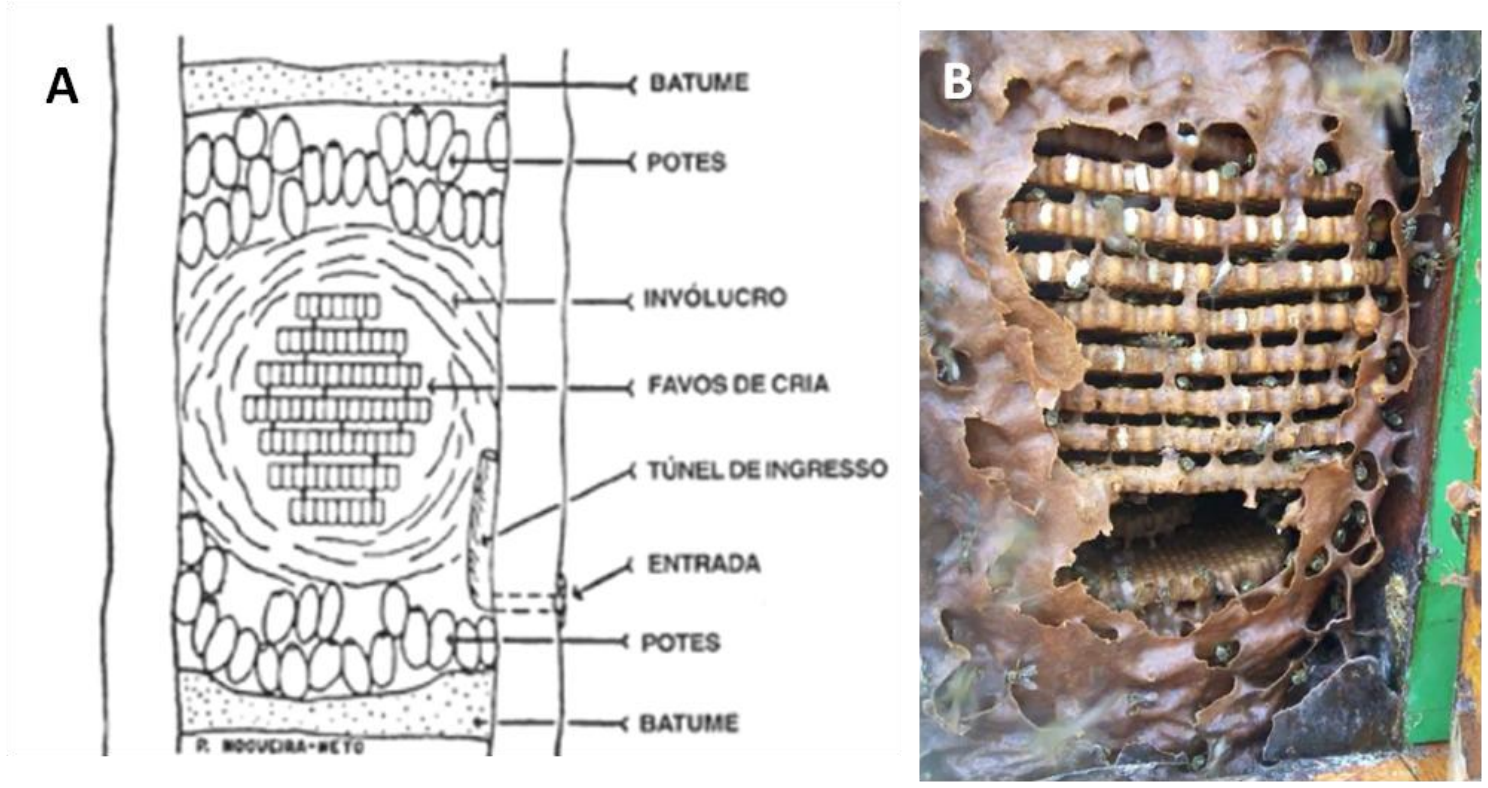
Figura 2. A. Esquema de um ninho de Meliponíneo de uma espécie que faz favos de cria compactos, horizontais ou helicoidais (NOGUEIRA-NETO, 1970) B. Imagem dos discos de cria da abelha sem ferrão Tetragona elongata Imagem: Gabriela T. de Paula

Ainda não foram descritos micro-organismos patogênicos específicos para as abelhas sem ferrão, principalmente devido à falta de trabalhos com esse foco envolvendo esses insetos (vanENGELSDORP e MEIXER, 2010). No entanto, múltiplos fatores estão envolvidos no declínio desses polinizadores, especialmente o desmatamento, que leva à perda de seu habitat natural ao reduzir a disponibilidade de locais adequados para a construção de seus ninhos e de recursos alimentares (PIOKER-HARA, 2014). A presença de abelhas exóticas, como Apis mellifera, também contribui para o declínio das abelhas nativas, devido a interações competitivas (NUNES-SILVA et al., 2016). Além disso, a intensificação agrícola aumenta a exposição das abelhas aos pesticidas (BARBOSA, 2015). Só em 2019 foram registrados um total de 474 agrotóxicos, a maior quantidade já registrada dos últimos 14 anos (MAPA, 2020). Dentre os agrotóxicos liberados estão incluídos o fipronil e os neonicotinóides imidaclopride e tiametoxam que são alguns dos inseticidas com comprovada toxicidade para algumas espécies de abelhas sem ferrão (TOMÉ, et al., 2012; LOURENÇO, et al., 2012; ROSA et al., 2016). Além disso, a presença de alguns fungicidas, como clorotalonil e piraclostrobina, no pólen de algumas plantas levou a um aumento da susceptibilidade de infecção pelo parasita intestinal Nosema spp. em abelhas melíferas e, com isso, a um comprometimento da saúde da colônia (PETTIS et al., 2013). Todos esses fatores podem acarretar sérias consequências para a polinização e reprodução de plantas nativas e cultivadas (OLIVEIRA et al., 1995; OLIVEIRA, 2001; DICK, 2001; BROWN e ALBRECHT, 2001).

Devido às recentes preocupações sobre o declínio das populações de abelhas e a diminuição concomitante da polinização, a microbiota simbionte das abelhas sociais e o seu significado para a saúde das colônias estão sendo objeto de intensos esforços para pesquisa (EVANS et al., 2006).

\subsection{Relações simbióticas de micro-organismos e outros hospedeiros}

A simbiose é definida como uma relação íntima entre organismos, usualmente entre diferentes espécies, muitas vezes de forma benéfica (WILKINSON, 2001; GILBERT et al., 2012; MARTIN e SCHWAB, 2013; McFALL-NGAI et al., 2013).

Um dos maiores exemplos de interação simbiótica é o ser humano, cujo trato gastrointestinal é colonizado por células microbianas que constituem cerca de dez vezes mais 
que as células do corpo humano (QIN et al., 2010). Essa relação simbiótica é mantida de forma bidirecional, nessa conformidade a microbiota intestinal se beneficia de um ambiente estável e rico em nutrientes, enquanto o hospedeiro usufrui da fermentação microbiana de componentes não-digeríveis pelo hospedeiro (fibras dietéticas), o que contribui para um ganho energético, além da produção de vitamina K e B12, e proteção contra potenciais patógenos (FLINT et al., 2012; CONLY et al., 1994; ALBERT et al., 1980; COLLADO et al., 2007; MARTIN et al., 2010; WOPEREIS et al., 2014).

Foi também demonstrado recentemente que bactérias simbióticas podem mediar os odores sociais das hienas. À medida que as bactérias fermentam ou metabolizam os substratos ricos em nutrientes presentes nas glândulas aromáticas das hienas, são gerados metabólitos voláteis com odores que, posteriormente, são usados por seus hospedeiros para comunicação (THEIS et al., 2013).

Os insetos sociais também são conhecidos por estabelecerem interação simbiótica com micro-organismos, pois são capazes de fornecer múltiplos habitats para o crescimento dos mesmos, como condições ambientais estáveis e uma rica fonte de alimento. Em troca, os micro-organismos podem proporcionar vários benefícios aos seus hospedeiros, como nutrientes e proteção contra patógenos (MUELLER et al., 2005; ROUBIK, 2006; ANDERSON et al., 2011; KALTENPOTH e ENGL, 2013). Um exemplo bastante conhecido envolve as formigas cortadeiras da tribo Attini, que cultivam um fungo pertencente à família Lepiotaceae (Basidiomycota: Agaricales), fornecendo-lhe condições ideais de crescimento. Em troca, o fungo serve como principal fonte de alimento, fornecendo lipídeos, carboidratos e aminoácidos essenciais para as larvas e formigas adultas (QUINLAN e CHERRETT, 1979; CURRIE, 2001; De FINE LICHT et al., 2014). De forma similar, cupins da subfamília Macrotermitinae cultivam um fungo pertencente ao gênero Termitomyces (Termitomyceteae: Tricholomataceae: Basidiomycota) que, além de ser a principal fonte de alimento, também fornece serviços digestivos (De FINE LICHT et al., 2005).

Recentemente foi descrita uma interação simbiótica entre a espécie de abelha sem ferrão Scaptotrigona depilis e o fungo Zygosaccharomyces sp. Esse fungo cresce no interior das células de cria de S. depilis e é ingerido pelas larvas (Figura 3), sendo indispensável para a sobrevivência e desenvolvimento das mesmas (MENEZES et al., 2015; PALUDO, 2017; PALUDO et al., 2018). 


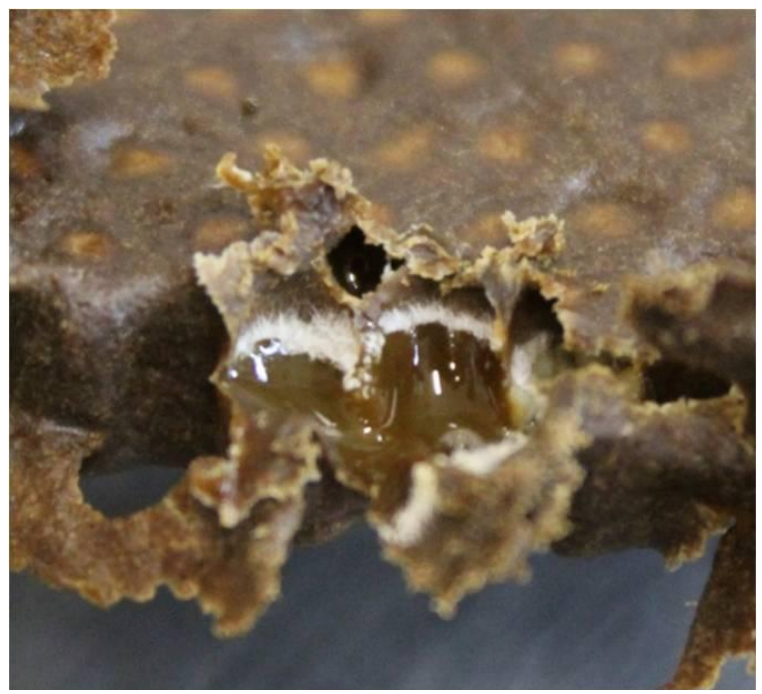

Figura 3. Células de cria de Scaptotrigona depilis com a presença do fungo-alimento Zygosaccharomyces sp. Imagem: Camila R. Paludo

Esse fungo-alimento trata-se de uma nova espécie de levedura osmofílica que é capaz de acumular lipídeos em adipossomos no seu citoplasma, dentre eles o esteroide ergosterol (PALUDO et al., 2018).

A metamorfose e o tamanho das abelhas são influenciados pela interação entre hormônio juvenil, ecdisteroides e sinalização de insulina/fator de crescimento semelhante à insulina (CHOLE, et al. 2019). Já se sabe que os insetos são altamente dependentes de fontes exógenas de esteroides que servem como precursores para a produção de ecdisteroides (ex.: 20-hidroxi-ecdisona), os quais atuam como hormônios de muda (BEHMER e NES, 2003). Esses ecdisteroides podem ser biossintetizados a partir de precursores esteroidais vegetais (Figura 4A) ou fúngicos (Figura 4B), dependendo da dieta do inseto. As larvas de S. depilis utilizam o esterol fúngico ergosterol para a produção dos ecdiesteroides makisterona A/24epi-makisterona (Figura 4B). Este foi o primeiro relato da descrição do fator nutricional/hormonal conferido por um fungo para o desenvolvimento de abelhas sem ferrão (PALUDO, 2017; PALUDO et al., 2018). 


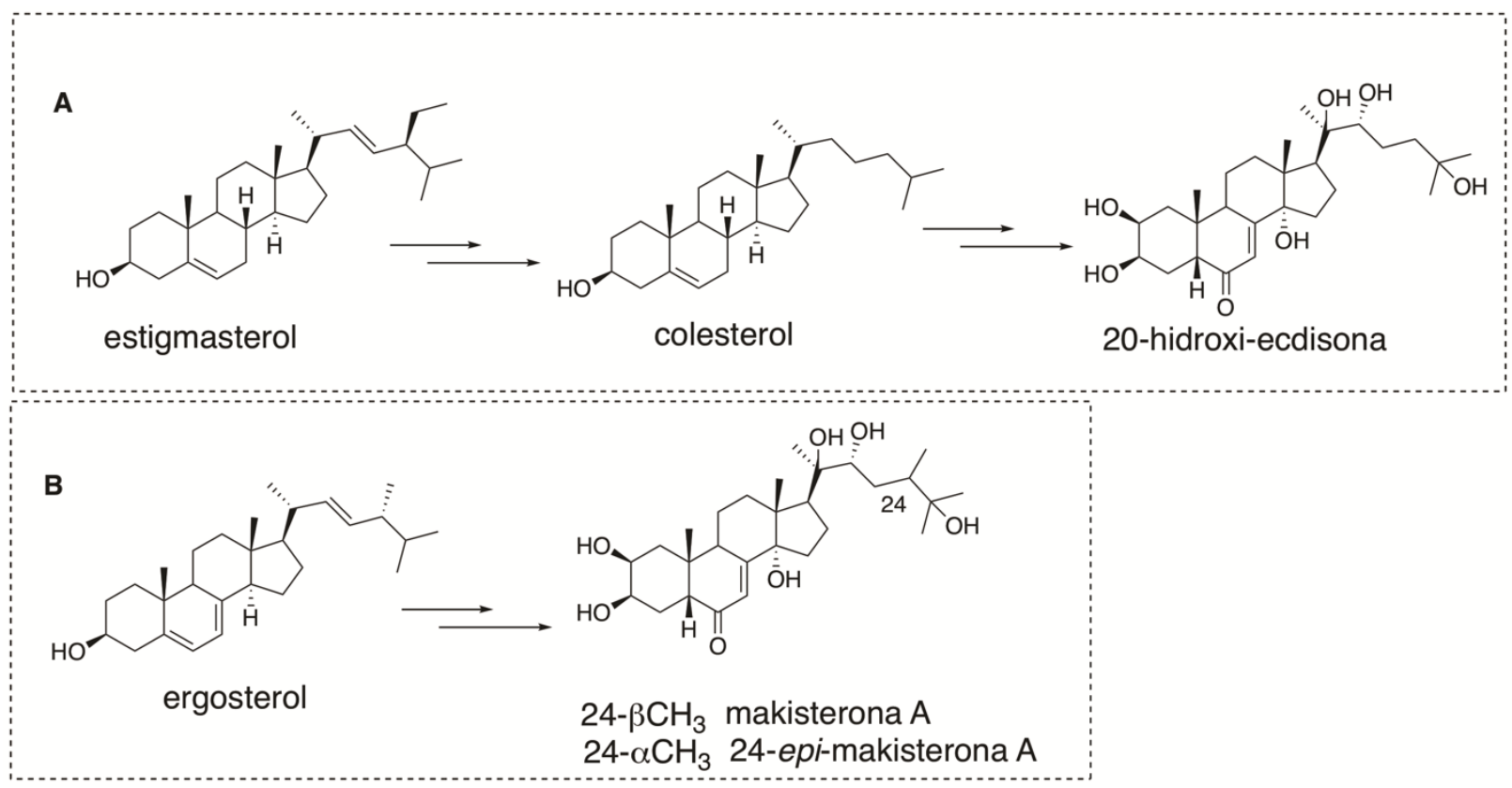

Figura 4. A. Produção do hormônio de muda 20-hidroxiecdisona em insetos a partir de precursor esteroidal vegetal (estigmasterol). B. Biossíntese do ecdisteroide makisterona A/24-epi-makisterona na abelha sem ferrão S. depilis, a partir do ergosterol (HUANG et al., 2008; PALUDO et al., 2018).

Complementarmente, também foi descrita a presença de outros fungos na célula de cria de S. depilis como Candida sp. e Monascus ruber, que interagem entre si e com Zygosaccharomyces sp., modulando o crescimento do fungo alimento através da produção de pequenas moléculas (PALUDO et al., 2019).

Apesar de já existirem alguns relatos do isolamento de Zygosaccharomyces em colônias de abelhas, não havia relatos na literatura sobre a importância desses micro-organismos para o ciclo de vida de abelhas sem ferrão, tampouco havia trabalhos esclarecendo e descrevendo o mecanismo molecular envolvido em tais interações simbióticas. Esse resultado, sem precedências na literatura, demonstra o quão intrincada pode ser a relação entre microorganismos e abelhas sem ferrão. Há uma grande variedade em espécies de abelhas sem ferrão no território brasileiro e, por essa razão, é de extrema importância determinar se essa simbiose nutritiva também está presente nesses meliponíneos.

Assim, a hipótese deste trabalho é que outras espécies de abelhas sem ferrão também apresentam fungos Zygosaccharomyces spp. em suas células de cria, que podem atuar como fontes de alimento pelo fornecimento de ergosterol, importante precursor biossintético de ecdisteroides. Os questionamentos decorrentes da hipótese da presença do fungo alimento são: os isolados de Zygosaccharomyces spp. das células de cria apresentam padrões diferenciados de morfologia e acúmulo de ergosterol em comparação com Zygosaccharomyces spp. isolados de outros locais das colônias? E quais as relações 
filogenéticas entre esses micro-organismos? É importante responder essas questões para entender a importância da microbiota no ciclo de vida das abelhas sem ferrão. Se fungo alimento é realmente importante para o desenvolvimento larval das abelhas sem ferrão, é de se preocupar que exista um grande uso de fungicidas na agricultura, com um total de 88 ingredientes ativos liberados (MAPA, 2020) que poderiam interferir indiretamente no declínio dessas abelhas. O entendimento do papel da microbiota das abelhas sociais pode aumentar a compreensão sobre o funcionamento desse sistema, com a geração de dados que contribuam para o delineamento de políticas públicas necessárias para sua preservação. 
Conclusões 


\section{CONCLUSÕES}

No presente trabalho foi possível verificar que o fungo Zygosaccharomyces spp. está comumente associado com as abelhas sem ferrão. Essa levedura está presente em diversas partes do ninho, tanto no mel, pólen, cerume, na abelha adulta, no refugo/lixo e principalmente nas células de cria. As linhagens de Zygosaccharomyces spp. isoladas das células de cria de diferentes espécies de abelhas sem ferrão formam um grupo específico que diverge filogeneticamente e morfologicamente das linhagens isoladas dos demais locais. Nesse grupo há uma variação nas espécies de Zygosaccharomyces de acordo com as espécies de abelhas sem ferrão em que se encontram, como observado nas análises filogenéticas, mas os mesmos compartilham de um ancestral em comum. São espécies novas ainda não descritas na literatura. Essas leveduras apresentam morfologia diferenciada das espécies de Zygosaccharomyces já descritas, como a formação de pseudo-hifas, o que sugere que essa condição seja, provavelmente, determinante para a presença desses fungos nesse ambiente específico. Devido às espécies pertencentes a esse grupo apresentarem semelhanças morfológicas e filogenéticas com a linhagem de Zygosaccharomyces descrita para o sistema envolvendo $S$. depilis, sugere-se que a principal função desses micro-organismos para as abelhas sem ferrão seja nutricional, como fonte de precursor esteroidal para a síntese de ecdisteroides, assim como já foi relatado para S. depilis.

Não foi possível inferir qual a função dos outros fungos isolados da célula de cria. Eventualmente esses diferentes fungos poderiam desempenhar o mesmo papel de Zygosaccharomyces ou modular o crescimento do mesmo. Diferentemente das espécies de Zygosaccharomyces, esses fungos são filamentosos e diferem filogeneticamente entre si. Provavelmente são espécies novas ainda não descritas na literatura. Espécies do gênero Monascus têm sido isoladas das células de cria de algumas espécies de abelhas sem ferrão, assim como descrito anteriormente para Scaptotrigona delipis (SDCP1), e agora para Scaptotrigona bipunctata (SBBCRP2).

Não foi observado um padrão diferenciado de concentração de ergosterol para as linhagens de Zygosaccharomyces spp. isoladas das células de cria em comparação com aquelas isoladas de diferentes partes da colônia e com outros fungos. Isso pode indicar que o recrutamento do micro-organismo pelas abelhas nesta associação simbiótica ocorre mais em função da adaptação do fungo para o crescimento neste microambiente de alta osmolaridade do que pela capacidade de acúmulo diferencial de ergosterol. Mais estudos são necessários para comprovar esta hipótese. 
O declínio das abelhas sem ferrão tem como causa vários fatores dentre eles o uso de agrotóxicos na agricultura. Já se sabe que o uso de pesticidas afeta diretamente as abelhas. Os resultados obtidos nesse trabalho permitem inferir que, devido à associação que há entre esses diferentes micro-organismos e as abelhas sem ferrão, o uso de fungicidas pode também afetar essa microbiota e com isso também prejudicar indiretamente as abelhas. Por isso, são necessários mais estudos que possam avaliar a toxicidade desses compostos nesse sistema. Esses estudos podem, no futuro, contribuir para o estabelecimento de normativas para o uso mais racional de agrotóxicos nas culturas agrícolas. 
Referências 


\section{REFERÊNCIAS}

AGUILERA, F.; PEINADO, R. A.; MILÁN, C.; ORTEGA, J. M. MAURICIO, J. C. Relationship between ethanol tolerance, $\mathrm{H}^{+}$-ATPase activity and the lipid composition of the plasma membrane in different wine yeast strains. International Journal of Food Microbiology, v. 110, p. 34-42, 2006. https://doi.org/10.1016/j.ijfoodmicro.2006.02.002

ALBERT, M. J.; MATHAN, V. I.; BAKER, S. J. Vitamin B12 synthesis by human small intestinal bacteria. Nature, v. 283, p. 781-782, 1980.

ANDERSON, K. E.; SHEEHAN, B. J.; ECKHOLM, B. M.; MOTT, G. An emerging paradigm of colony health: microbial balance of the honey bee and hive (Apis mellifera). Insectes Sociaux, v. 58, p. 431-444, 2011.

ARX, J.; MIRANDA, R.; SMITH, M.; YARROW, D. The genera of the yeasts and the yeastlike fungi. Stud Mycol, v. 14, p. 1-42, 1977.

ASCHER, J. S. \& PICKERING, J. Discover Life bee species guide and world checklist (Hymenoptera: Apoidea: Anthophila), 2020. Disponível em: http://www.discoverlife.org/mp/20q?guide=Apoidea_species. Acesso em: 15 fev. 2020.

BARBOSA, W. F.; SMAGGHE, G.; GUEDES, R. N. C. Pesticides and reduce-risk insecticides, native bees and pantropical stingless bee: pitfalls and perspectives. Pest Management Science, v. 71, p. 1049-1053, 2015.

BARBOSA, R. N.; LEONG, S. L.; VINNERE-PETTERSSON, O.; CHEN, A. J.; SOUZAMOTTA, C.M.; FRISVAD, J. C.; SAMSON, R.A.; OLIVEIRA, N.T.; HOUBRAKEN, J. Phylogenetic analysis of Monascus and new species from honey, pollen and nests of stingless bees. Studies in Mycology, v. 86, p. 29-51, 2017. https://doi.org/10.1016/j.simyco.2017.04.001

BEHMER, S. T.; NES, W. D. Insect sterol nutrition and physiology: a global overview. In Advances in insect physiology, S. J. Simpson, ed. (Elsevier), v. 31, pp. 1-72, 2003.

BLOCH, K. E. Sterol, Structure and Membrane Function. Critical Reviews in Biochemistry, v. 14(1), p. 47-92, 1983. doi:10.3109/10409238309102790

BREIVIK, O. N.; OWADES, J. L. Yeast analysis, spectrophotometric semimicrodetermination of ergosterol in yeast. Journal of Agricultural and Food Chemistry, v. 5 (5), p. 360-363, 1957. doi: 10.1021/jf60075a005

BRYSCH-HERZBERG, M.; WOHLMANN, E.; FISCHER, R. Zygosaccharomyces seidelii sp. nov. a new yeast species from the Maldives, and a revisit of the singles-strain species debate. Antonie van Leeuwenhoek, v. 113, p. 427-436, 2020. https://doi.org/10.1007/s10482-019-01352-X

BROWN, J. C.; ALBRECHT, C. The effect of tropical deforestation on stingless bees of the genus Melipona (Insecta: Hymenoptera: Apidae: Meliponini) in central Rondonia, Brazil. Journal of Biogeography, v. 28, p. 623-634, 2001. 
CADEZ, N.; FULOP, L.; DLAUCHHY, D.; PÉTER, G. Zygosaccharomyces favi sp. nov., an obligate osmophilic yeast species from bee bread and honey. Antonie Van Leeuwenhoek, v. 107, p. 645- 654, 2015.

CAMARGO, J. M. F.; PEDRO, S. R. M. Meliponini Lepeletier, 1836. In: MOURE, J. S.; URBAN, D.; GAR, M. (eds) Catalogue of bees (Hymenoptera, Apoidea) in the neotropical region - online version, 2013. Disponível em: http://www.moure.cria.org.br/catalogue. Acesso em : 15 fev. 2020.

CARVAlHO, C. M.; MEIRINHO, S.; ESTEVINHO, M. L. F.; CHOUPINA, A. Yeast species associated with honey: different identification methods. Archivos de zootecnia, v. 59, p. 103-113, 2010.

CHOLE, H.; WOODARD, S. H.; BLOCH, G. Body size variation in bess: regulation, mechanisms, and relationship to social organization. Current Opinion in Insect Science, v. 35, p. 77-87, 2019.

COLLADO, M. C.; DERRIEN, M.; ISOLAURI, E.; de VOS, W. M.; SALMINEN, S. Intestinal integrity and Akkermansia muciniphila, a mucindegrading member of the intestinal microbiota present in infants, adults, and the elderly. Applied and Environmental Microbiology, v. 73, p. 7767-7770, 2007.

CONLY, J. M.; STEIN, K.; WOROBETZ, L.; RUTLEDGE, H. S. The contribution of vitamin K2 (menaquinones) produced by the intestinal microflora to human nutritional requirements for vitamin K. The American Journal of Gastroenterology, v. 89, p. 915923, 1994.

CURRIE, C. R. A community of ants, fungi, and bacteria: A multilateral approach to studying symbiosis. Annual Review of Microbiology, v. 55, p. 357-380, 2001.

DANFORTH, B. Bees. Currenty Biology, v. 17(5), p. 156-161, 2007.

De FINE LICHT, H.H.; ANDERSEN, A.; AANEN, D. K. Termitomyces sp. associated with the termite Macrotermes natalensis has a heterothallic mating system and multinucleate cells. Mycological Research, v. 109 (3), p. 314-318, 2005.

De FINE LICHT, H. H.; BOOMSMA, J. J.; TUNLID, A. Symbiotic adaptations in the fungal cultivar of leaf-cutting ants. Nature Communications, v. 5, e5675, 2014. DOI:10.1038/ncomms6675.

DEWICK, P.M. Medicinal Natural Products: A Biosynthetic Approach. Chichester: John Wiley \& Sons, UK, 3 ed., p. 539, 2009.

DICK, C. W. Habitat change, African honeybees, and fecundity in the Amazonian tree Dinizia excelta (Fabaceae). In: BIERREGAARD Jr., R. O.; GASCON, C.; LOVEJOY, T. E.; MESQUITA, R. (eds.) Lesson from Amazonia: the ecology and conservation of a fragmented forest. Yale University Press, New Haven, p. 146-157, 2001.

EVANS, J.D.; ARONSTEIN, K.; CHEN, Y. P.; HETRU, C.; IMLER, J. L.; JIANG, H.; KANOST, M.; THOMPSON, G. J.; ZOU, Z.; HULTMARK, D. Immune pathways and 
defence mechanisms in honeybees Apis mellifera . Insect Molecular Biology, v. 15, p. 645656, 2006.

FLINT, H.J.; SCOTT, K. P.; LOUIS, P.; DUNCAN, S. H. The role of the gut microbiota in nutrition and health. Nature Reviews Gastroenterology Hepatology, v. 9, p. 577-589, 2012.

GILBERT, S. F.; SAPP, J.; TAUBER, A. I. A symbiotic view of life: We have never been individuals. The Quarterly Review of Biology, v. 87(4), p. 325-341, 2012.

HABIB, H. M.; AL MEQBALI, F. T.; KAMAL, H.; SOUKA, U. D.; IBRAHIM, W. H. Physicochemical and biochemical properties of honey from arid regions. Food Chemistry, v. 153, p. 35-43, 2014.

HALL, T.A. BioEdit: a user-friendly biological sequence alignment editor and analysis program for Windows 95/98/NT. Nucleic Acids Symposium Series, v. 41,p. 95-98, 1999.

HARTFELDER, K.; ENGELS, W. The composition of larval food in stingless bees: evaluating nutritional balance by chemosystematic methods. Insectes Sociaux, v. 36, p. 1-14, 1989.

HÖLLDOBLER, B.; WILSON, E.O. The superorganism: the beauty, elegance, and strangeness of insect societies. WW Norton \& Company, 2009

HOICZYK, E.; RING, M.W.; MCHUGH, C. A.; SCHW ÄR, G.; BODE, E.; KRUG, D.; ALTMEYER, M. O.; LU, J. Z.; BODE, H. B. Lipid body formation plays a central role in cell fate determination during developmental differentiation of Myxococcus xanthus. Molecular Microbiology, v. 74, p. 497-517, 2009.

HU, Z.; HE, B.; MA, L.; SUN, Y.; NIU, Y.; ZENG, B. Recent Advances in Ergosterol Biosynthesis and Regulation Mechanisms in Saccharomyces cerevisiae. Indian journal of microbiology, v. 57, 270-277, 2017. https://doi.org/10.1007/s12088-017-0657-1

HUANG, X., WARREN, J. T.; GILBERT, L. I. New players in the regulation of ecdysone biosynthesis. Journal of Genetics and Genomics, v. 35, p. 1-10, 2008.

HULIN, M.; WHEALS, A. Rapid identification of Zygosaccharomyces with genus-specific primers. International Journal of Food Microbiology, v. 173, p. 9-13, 2014.

JAMES, S. A.; STRATFORD, M. Zygosaccharomyces Barker (1901). In: KURTZMAN, C. P.; FELL, J. W.; BOEKHOUT, T. (Ed.) The Yeasts, a taxonomic study, Elsevier, 5 ed., v. 2, p. 937-947, 2011.

JIANG, Y. W.; KANG, C. M. Induction of S. cerevisiae filamentous differentiation by slowed DNA synthesis involves Mec1, Rad53 and Swe1 checkpoint proteins. Molecular Biology of the Cell, v. 14, p. 5116-5124, 2003.

KALTENPOTH, M.; ENGL, T. Defensive microbial symbionts in Hymenoptera. Functional Ecology, v. 28, p. 315-327, 2013. 
KERR, W. E.; CARVAlHO, G. A.; NASCIMENTO, V. A. Abelha Uruçu: Biologia, Manejo e Conservação. Belo Horizonte, MG: Acangaú, 1996, 154 p.

KIM, J.; ROSE, M. D. Stable pseudohyphal growth in budding yeast induce by synergism between septin defects and altered MAP-kinase signaling. PLoS Genetics, 11(12): e1005684, 2015. https://doi.org/10.1371/journal.pgen.1005684

KUMAR, S.; STECHER, G.; TAMURA, K. MEGA7: Molecular Evolutionary Genetics Analyses verson 7.0 for bigger datasets. Molecular Biology and Evolution, v.33, p. 18701874, 2016.

KURTZMAN, C. P.; ROBNETT, C. J. Identification and phylogeny of ascomycetous yeasts from analysis of nuclear large subunit (26S) ribosomal DNA partial sequences. Antonie Van Leeuwenhoek, Amsterdam, v. 73, n. 4, p. 331- 371, 1998.

KURTZMAN, C. P.; FELL, J. W.; BOEKHOUT, T.; ROBERT, V. Methods for isolation, phenotypic characterization and maintenance of yeasts. In: KURTZMAN, C. P.; FELL, J. W.; BOEKHOUT, T. (Ed.) The Yeasts, a taxanomic study, Elsevier, 5 ed., v. 1, p. 87-110, 2011.

LO, W. S.; DRANGINIS, A. M. The cell surface flocculin FLO11 is required for pseudohiphae formation and invasion by Saccharomyces cerevisiae. Molecular Biology of the Cell, v. 9, p. 161-171, 1998.

LORENZ, M. C.; CUTLER, N. S.; HEITMAN, J. Characterization of alcohol-induced filamentous growth in Saccharomyces cerevisiae. Molecular Biology of the Cell, v. 11, p. 183-199, 2000.

LOURENÇO, C. T.; CARVALHO, S. M.; MALASPINA, O.; NOCELLI, R. C. F. Oral toxicity of fipronil insecticide against the stingless bee Melipona scutellaris (Letreille, 1811). Bulletin of Environmental Contamination and Toxicology, v. 89, p. 921-924, 2012. https:/doi.org/10.1007/s00128-012-0773-X

MARTIN, R.; NAUTA, A.; BEM, A. K.; KNIPPELS, L.; KNOL, J.; GARSSEN, J. Early life: gut microbiota and immune development in infancy. Beneficial Microbes, v. 1, p. 367$382,2010$.

MARTIN, B. D.; SCHWAB, E. Current usage of symbiosis and associated terminology. International Journal of Biology, v. 5, p. 32-45, 2013.

MATOS, T. T. S.; TEIXEIRA, J. F.; MACIAS, L. G.; SANTOS, A. R. O; SUH, S. O.; BARRIO, E.; LACHANCE, M. A.; ROSA, C. A. Kluyveromyces osmophilus is not a synonym of Zygosaccharomyces mellis; reinstatement as Zygosaccharomyces osmophilus comb. nov. International Journal of Systematic and Evolutionary Microbiology, v. 70, p. 3374-3378, 2020. <https://doi.org/10.1099/ijsem.0.004182>

McFALL-NGAI, M., HADFIELD, M. G.; BOSCH, T. C. G.; CAREY, H. V.; DOMAZETLOŠO, T.; DOUGLAS, A. E.; DUBILIER, N.; EBERL, G.; FUKAMI, T.; GILBERT, S.F.; HENTSCHEL, U.; KING, N.; KJELLEBERG, S.; KNOLL, A. H.; KREMER, N.; MAZMANIAN, S. K.; METCALF, J. L.; NEALSON, K.; PIERCE, N. E.; RAWLS, J. F.; REID, A.; RUBY, E. G.; RUMPHO, M.; SANDERS, J. G.; TAUTZ, D.; WERNEGREEN, 
J. J. Animals in a bacterial world, a new imperative for the life sciences. Proceedings of the National Academy of Sciences of the United States of America, v. 110(9), p. 3229-3236, 2013.

MENEZES, C.; VOLLET-NETO, A.; MARSAIOLI, A. J.; ZAMPIERI, D. FONTOURA, I. C.; LUCHESSI, A. D.; IMPERATRIZ-FONSECA, V. L. A Brazilian social bee must cultivate fungus to survive. Current Biology, v. 25, p. 2851-2855, 2015.

MICHENER, C. D. The Bees of the World. Baltimore: The Johns Hopkins University Press, USA, 2 ed., 2007, 953 p.

MICHENER, C. D. The Meliponini. In: VIT, P.; PEDRO, S.R.M.; ROUBIK, D.W. (Eds) Pot-Honey: A legacy of stingless bees. New York: Springer DOI: 10.1007/978-1-46144960-7_40.2013, p. 3-18.

MUELLER, U. G.; GERARDO, N. M.; AANEN, D. K.; SIX, D. L.; SCHULTZ, T. R. The evolution of agriculture in insects. Annual Review of Ecology, Evolution and Systematics, v. 36, p. 563-595, 2005.

NOGUEIRA-NETO, P. Vida e Criação de Abelhas indígenas sem ferrão. $2^{\mathrm{a}}$ ed. São Paulo: Editora Tecnapis, $1970,50 \mathrm{p}$.

NOGUEIRA-NETO, P. Vida e Criação de Abelhas indígenas sem ferrão. São Paulo: Editora Nogueirapis, 1997, 445 p.

NUNES-SILVA, P.; PIOT, N.;MEEUS, I.;BLOCHTEIN, B.; SMAGGHE, G. Absence of Leishmaniinae and Nosematidae in stingless bees. Scientific Reports, DOI: 10.1038/srep32547, 2016.

OLIVEIRA, M. L.; MORATO, E. F.; GARCIA, M. V. B. Diversidade de espécies e densidade de ninhos de abelhas sociais sem ferrão (Hymenoptera, Apidae, Meliponinae) em floresta de terra firme na amazônia central. Revista Brasileira de Zoologia, v. 12, p. 13-24, 1995.

OLIVEIRA, M. L. Stingless bees (Meliponini) and orchid bees (Euglossini) in "terra firme" tropical forests and forest fragments. In: BIERREGAARD Jr., R. O.; GASCON, C.; LOVEJOY, T. E.; MESQUITA, R. (eds.) Lessons from Amazonia: the ecology and conservation of a fragmented forest. Yale University Press, New Haven, p. 208-218, 2001.

OLIVEIRA, E. C.; MULLER, E. I.; ABAD, F.; DALLAROSA, J.; ADRIANO, C. Internal standard versus external standard calibration: an uncertainly case study of a liquid chromatography analysis. Química Nova, v. 33, n. 4, p. 984-987, 2010. <https://doi.org/10.1590/S0100-40422010000400041>

PALUDO, C. R. Estudo químico e biológico de micro-organismos associados à abelha sem ferrão Scaptotrigona depilis. 2017, 173 p. Tese de Doutorado, apresentada à Faculdade de Ciências Farmacêuticas de Ribeirão Preto/USP - Área de concentração: Produtos Naturais e Sintéticos, Ribeirão Preto, 2017.

PALUDO, C. R.; MENEZES, C.; SILVA-JUNIOR, E. A.; VOLLET-NETO, A.; ANDRADE-DOMINGUEZ, A.; PISHCHANY, G.; KHADEMPOUR, L.; NASCIMENTO, F. S.; CURRIE, C. R.; KOLTER, R.; CLARDY, J.; PUPO, M. T. Stingless Bee Larvae 
Require Fungal Steroid to Pupate. Scientific Reports, v. 8, e1122, 2018. DOI: 10.1038/s41598-018-19583-9.

PALUDO, C. R.; PISHCHANY, G.; ANDRADE-DOMINGUES, A.; SILVA-JÚNIOR, E. A.; MENEZES, C.; NASCIMENTO, F. S.; CURRIE, C. R.; KOLTER, R.; CLARDY, J.; PUPO, M. T. Microbial community modulates growth of symbiotic fungus required for stingless bee metamorphosis. PLoS ONE, 14(7): e0219696, 2019. https://doi.org/10.1371/journal.pone.0219696

PETTIS, J. S.; LICHTENBERG, E. M.; ANDREE, M.; STITZINGER, J.; ROSE, R.; vanENGELSDORP, D. Crop pollination exposes honey bees to pesticides which alters their susceptibility to the gut pathogen Nosema ceranae. PLoS ONE, 8(7): e70182, 2013. https://doi.org./10.1371/journal.pone.0070182

PIOKER-HARA, F. C.; DRUMMOND, M. S.; KLEINERT, A. D. M. P. The influence od the loss of Brazilian savanna vegetation on the occurrence of nests of stingless bess (Apidae: Meliponini). Sociobiology, v. 61, p. 393-400, 2014.

PIIRONEN, V.; LINDSAY, D. G.; MIETTINEN, T. A.; TOIVO, J. LAMPI, A. M. Plant sterols: biosynthesis, biological function and their importance to human nutrition. Journal of the Science of Food and Agriculture, v. 80, p. 939-966, 2000.

QIN, J.; LI, R.; RAES, J.; et al. A human gut microbial gene catalogue established by metagenomic sequencing. Nature, v. 464, p. 59-65, 2010.

QUINLAN, R. J. \& CHERRETT, J. M. The role of fungus in the diet of the leaf-cutting ant Atta cephalotes (L.). Ecological Entomology, v.4, p. 151-160, 1979.

RASMUSSEN, C. \& CAMERON, S. A. Global stingless bee phylogeny supports ancient divergence, vicariance, and long distance dispersal. Biological Journal of the Linnean Society, v. 99, p. 206-232, 2010.

RODRIGUEZ, R. J.; LOW, C.; BOTTEMA, C. D. K.; PARKS, L. W. Multiple functions for sterols in Saccharomyces cerevisiae. Biochimica et Biophysica Acta (BBA) - Lipids and Lipid Metabolism, v. 837(3), p. 336-343, 1985. doi:10.1016/0005-2760(85)90057-8

ROSA, C. A.; LACHANCE, M. A. Zygosaccharomyces machadoi sp. n., a yeast species isolated from a nest of the stingless bee Tetragonisca angustula. Lundiana, v. 6, p. 27-29, 2005 .

ROSA, A. D. S.; TEIXEIRA, J. S. G.; VOLLET-NETO, A.; QUEIROZ, E. P.; BLOCHTEIN, B.; PIRES, C. S. S.; IMPERATRIZ-FONSECA, V. L. Consumption of the neonicotinoid thiamethoxam during the larval stage affects the survival and development of the stingless bee, Scaptotrigona aff. depilis. Apidologie, v. 47, p. 729-738, 2016. https://doi.org/10.1007/s13592-015-0424-4

ROSTRON, K. A.; ROLPH, C. E.; LAWRENCE, C. L. Nile red fluorescence screening facilitating neutral lipid phenotype determination in budding yeast, Saccharomyces cerevisiae, and the fission yeast Schizosaccharomyces pombe. Antonie van Leeuwenhoek, v. 108, p. 97-106, 2015. doi:10.1007/s10482-015-0467-6

ROUBIK, D. W. Stingless bee nesting biology. Apidologie, v. 37, p. 124-143, 2006. 
SAKAGAMI, S. F. Stingless Bees. In: Social Insects. Vol. III (Hermann HR, ed.). New York: Academic Press, p. 361-423, 1982.

SAKAGAMI, S. F..; ROUBIK, D. W.; ZUCCHI R. Ethology of the robber stingless bee, Lestrimelitta limao (Himenoptera: Aipidae). Sociobiology, v.21, p. 237-277, 1993.

SAKSINCHAI, S.; SUZUKI, M.; CHANTAWANNAKUL, P.; OHKUMA, M.; LUMYONG, S. A novel ascosporogenous yeast species, Zygosaccharomyces siamensis, and the sugar tolerant yeasts associated with raw honey collected in Thailand. Fungal Divers, v. 52, p. 123-139, 2012.

SHARMA, A. D.; SINGH, J. A nonenzymatic method to isolate genomic DNA from bacterial and actinomycete. Analytical biochemistry, v. 337, n. 2, p. 354-356, 2005.

SCHOCH, C. L.; SEIFERT, K. A.; HUHNDORF, S.; ROBERT, V.; SPOUGE, J. L.; LEVESQUE, C. A.; CHEN, W. Huhndorf S, Robert V, Spouge, JL, Levesque CA, Chen W, et al. Nuclear ribosomal internal transcribed spacer (ITS) region as a universal DNA barcode marker for fungi. Proc Natl Acad Sci, v. 109, p. 6241-6246, 2012.

SILVEIRA, F. A.; MELO, G. A. R.; ALMEIDA, E. A. B. Abelhas brasileiras: sistemática e identificação. Belo Horizonte, p. 253, 2002.

SLAA, E. J.; SÁNCHEZ CHAVES, L. A.; MALAGODI-BRAGA, K. S.; HOFSTEDE, F. E. Stingless bees in applied pollination: practice and perspectives. Apidologie, v. 37 (2), p. 293 315, 2006.

SOLIERI, L.; DAKAL, T. C.; GIUDICI, P. Zygosaccharomyces sapae sp. nov., isolated from Italian traditional balsamic vinegar. International Journal of Systematic and Evolutionary Microbiology, v. 63, p. 364-371, 2013.

SUH, S. O.; GUJJARI, P.; BERES, C.; BECK, B.; ZHOU, J. Proposal of Zygosaccharomyces parabailii sp. nov. and Zygosaccharomyces pseudobailii sp. nov., novel species closely related to Zygosaccharomyces bailii. International Journal of Systematic and Evolutionary Microbiology, v. 63, p. 1922-1929, 2013.

THEIS, K. R.; VENKATARAMAN, A.; DYCUS, J. A.; KOONTER, K. D.; SCHMITTMATZEN, E. N.; WAGNER, A. P.; HOLEKAMP, K. E.; SCHMIDT, T. M. Symbiotic bacteria appear to mediate hyena social odors. Proceedings of the National Academy of Sciences of the United States of America, v. 110, p.19832-19837, 2013.

TOMÉ, H. V.; MARTINS, G. F.; LIMA, M. A.; CAMPOS, L. A.; GUEDES, R. N. Imidacloprid-induced impairment of mushroom bodies and behavior of the native stingless bee Melipona quadrifasciata anthidioides. PLoS One, v. 7(6):e38406, 2012. doi:10.1371/journal.pone.0038406

TORRIANI, S.; LORENZINI, M.; SALVETTI, E.; FELIS, G. E. Zygosaccharomyces gambellarensis sp. nov., an ascosporogenous yeast isolated from an Italian 'passito'style wine. International Journal of Systematic and Evolutionary Microbiology, v. 61, p. 3084-3088, 2011. 
VAN ENGELSDORP, D.; MEIXNER, M. D. A historical review of managed honey bee populations in Europe and the United States and the factors that may affect them. Journal of Invertebrate Pathology, v. 63, p. S80-S95, 2010.

VELTHUIS, H. H. W.; VRIES, H. D.; IMPERATRIZ-FONSECA, V. L. The polygyny of Melipona bicolor: scramble competition among queens. Apidologie, v. 37, p. 222239, 2006.

VENTURIERI, G. C. Criação de abelhas indígenas sem ferrão. 2a Edição. Belém-PA: Embrapa Amazônia Oriental, 2008.

WILKINSON, D. M. At cross purposes. Nature, v. 412, p. 485, 2001.

WILSON, E. O. Success and dominance in ecosystems: the case of the social insects. Ecology Institute, Oldendorf, p. 1990.

WOPEREIS, H.; OOZEER, R.; KNIPPING,K.; BELZER, C.; KNOL, J. The first thousand days - intestinal microbiology of early life: establishing a symbiosis. Pediatric Allergy Immunolog, v. 25, p. 428-438, 2014. 